\title{
Spontaneous Perforation of Gallbladder: Case Report
}

\author{
Satish Kumar Sheoran a, b, Rajiv Nandan Sahai ${ }^{a}$, Jagmohan Indora ${ }^{a}$, \\ Upender Chand Biswal ${ }^{\mathrm{a}}$
}

\begin{abstract}
The main cause of perforation of the gallbladder is cholecystitis with or without cholelithiasis. In old age, spontaneous perforation of gallbladder can be due to decrease in its blood supply, which can be due to atherosclerosis, focal vasospasm or localized vasculitis. Perforation of gallbladder is associated with high morbidity and mortality, if left untreated. Here we report a case of a 60 -year-old male with perforation of gallbladder.
\end{abstract}

Keywords: Spontaneous perforation; Gallbladder

\section{Introduction}

The main cause of gallbladder perforation is cholecystitis with or without gallstones [1]. The reported incidence of gallbladder perforation in acute cholecystitis is $2-18 \%$ [2]. However, cases reported as idiopathic or spontaneous gallbladder perforation are not only rare but also have features that are different from those occurring as a complication of cholecystitis. Their different features can be described as peritonitis caused by gallbladder perforation lacking the typical clinical presentation, radiological and histopathological characteristics of cholecystitis or gallbladder perforation [3]. As a result, the diagnosis is often delayed or even missed.

\section{Case Report}

A 60-year-old male presented in surgical emergency with pain in upper abdomen for 5 days with increase in its intensity over the last $4 \mathrm{~h}$. The pain was more in the epigastrium and right

Manuscript accepted for publication May 16, 2016

a'Department of Surgery, NDMC Medical College \& Hindu Rao Hospital, Delhi, India

${ }^{b}$ Corresponding Author: Satish Kumar Sheoran, Department of Surgery, NDMC Medical College \& Hindu Rao Hospital, 2/19, Geeta Colony, Delhi 110031, India. Email: satishsheoran08@gmail.com

doi: http://dx.doi.org/10.14740/gr702e hypochondrium with nausea, occasional vomiting and upper abdominal distention. It was also accompanied by non-passage of flatus and feces for 1 day. The stool was clay-colored and there was yellowish discoloration of urine for last 2 days. He was a chronic smoker and alcoholic. There was no history of any other comorbidity. His BP was $110 / 60 \mathrm{~mm} \mathrm{Hg}$, PR was $120 / \mathrm{min}$, and he had icterus. On abdominal examination, it was found distended and tender in upper half with guarding. His routine investigations revealed his $\mathrm{Hb}$ was $10.7 \mathrm{~g}$, TLC was 26,800 , T. bilirubin was 6.0 (direct 4.0 ), serum urea was 90 , s. creatinine was 2.2. PT, INR, amylase and lipase were within normal limits. X-ray abdomen erect did not show any free gas under the right dome of diaphragm.

USG abdomen showed 1) a large anechoic collection with internal echoes and thin septations in perihepatic space of the size of $114 \times 81 \times 101 \mathrm{~mm}$, volume of $492 \mathrm{~mL}, 2$ ) another collection in subhepatic space of size $98 \times 85 \times 66 \mathrm{~mm}$, volume $286 \mathrm{~mL}, 3)$ non-visualization of gallbladder and 4) no abnormality of rest of the viscera are. A provisional diagnosis of ruptured liver abscess was made and exploratory laparotomy was done.

The following were the operative finding: upper abdomen contained about $1 \mathrm{~L}$ of bile stained fluid. There was a big rent in the fundal area of gallbladder (Fig. 1). No gallstone was found in peritoneal cavity.

Cholecystectomy was performed followed by thorough peritoneal lavage and a subhepatic drain was placed. In postoperative period, there was about $200 \mathrm{~mL}$ bilious drainage daily from subhepatic drain. On fifth postoperative day, the patient complained of pain abdomen with distention and there was biliary soakage of main laparotomy wound dressing.

MRCP was done which showed a large filling defect at the lower end of the common bile duct (CBD) and minor leak from the cystic duct stump (Fig. 2).

Following this, ERCP was done and a stone of about 10 $12 \mathrm{~mm}$ was removed from the CBD after fragmentation with lithotripsy. The patient recovered fully subsequently and discharged on the 10th postoperative day.

\section{Discussion}

Gallbladder perforation is a rare but life-threatening event [4]. In 1934, Niemeier [5] proposed a classification of gallbladder 


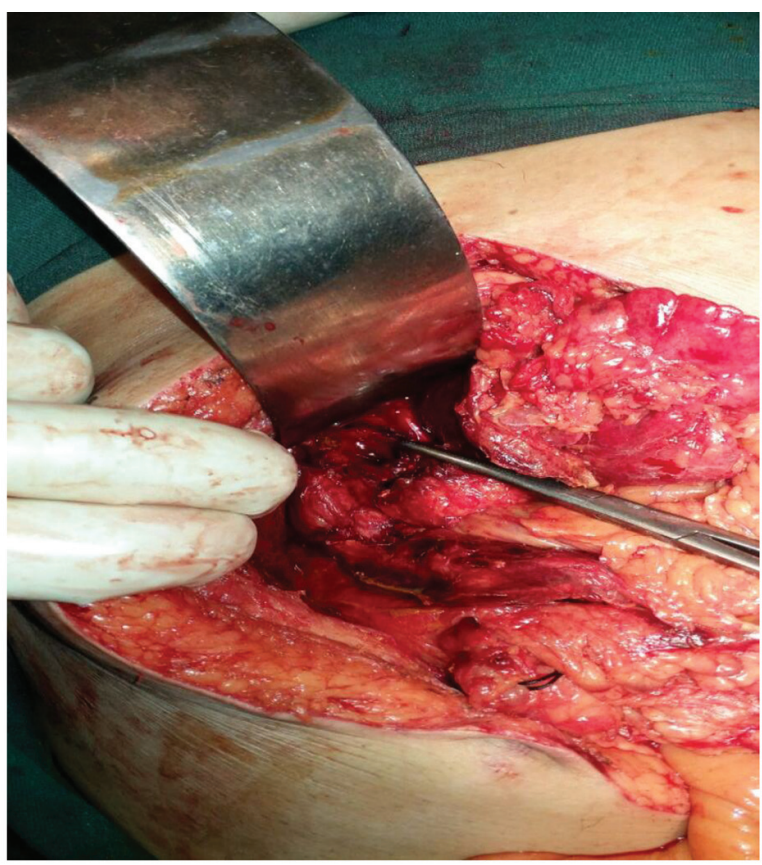

Figure 1. Perforation of gallbladder at fundus.

perforation: type 1 - acute free perforation into peritoneal cavity; type 2 - subacute perforation with pericholecystic abscess; and type 3 - chronic perforation with cholecystoenteric fistula. A number of modifications have been proposed, but the basic classification still stands. Roslyn et al [6] reported in his study that type 1 and type 2 gallbladder perforations are mostly seen in young patients ( $<50$ years), and type 3 is seen in elderly with long history of stone disease. Our patient had a type 2 gallbladder perforation. Estevao Costa et al [3] insisted that almost all cases of gallbladder perforation were in fact secondary to a coexistent disease such as inflammation, trauma or obstruction. They also proposed a classification system, which categorized the perforation of gallbladder into three groups: 1) spontaneous; 2) traumatic; and 3) iatrogenic. The spontaneous group is further sub-divided into an idiopathic and a secondary group, which includes acute inflammation, infection, lithiasis, congenital obstruction and anticoagulant therapy. Although there is little consensus about the classification, the most possible mechanism of gallbladder perforation in acute cholecystitis is cystic duct obstruction (mostly by a stone at the neck) that causes retention of intraluminal secretions leading to rise in intraluminal pressure. This raised intraluminal pressure compromises the venous and lymphatic drainage of gallbladder resulting in necrosis and finally gallbladder perforation [7]. As gallbladder perforation occurs most commonly at fundus due to least blood supply ( $60 \%$ of the cases in the study of Derici et al) [4], this proves the importance of ischemic mechanism. Conditions such as cholelithiasis, infections, malignancy, diabetes, atherosclerosis, steroid therapy, etc. are all predisposing risk factors for gallbladder perforation [2].

Clinical diagnosis of spontaneous gallbladder perforation is very difficult and often delayed or missed because there are no classical symptoms and signs of gall bladder perforation.

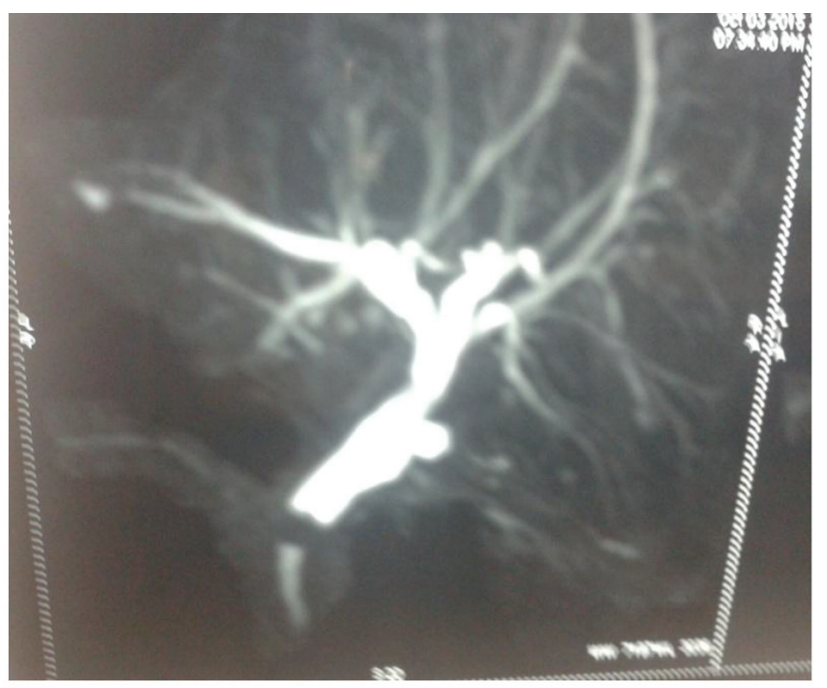

Figure 2. MRCP showing filling defect (stone) at distal end of CBD and minor leak from cystic duct stump.

It is difficult to discriminate clinically between the patients with perforated gallbladder and those with uncomplicated acute cholecystitis. A sudden decrease in pain intensity caused by the relief of high intracholecystic pressure might herald the perforation according to Chen et al [8]. Perforation and abscess formation should be suspected in those patients with acute cholecystitis who suddenly become toxic and whose clinical condition deteriorates rapidly as suggested by Gore et al [9]. Ultrasonography is the initial radiological investigation done in most of the cases but finding are very nonspecific for gallbladder perforation and mimic those seen in acute uncomplicated cholecystitis such as gallbladder distention (largest diameter $>3.5-4.0 \mathrm{~cm}$ ), gallbladder wall thickening (>3 mm), pericholecystic fluid collection, gallstones, coarse intracholecystic echogenic debris and bile duct dilatation. Distention of the gallbladder and edema of its wall may be the earliest detectable signs of imminent perforation. The hole sign [10] (a defect in the gallbladder wall) is the most specific finding. Solva et al [11] showed in their study that distention of gallbladder and edema of its wall may be earliest sign of impending perforation on ultrasonography. Computed tomography (CT) scan is the most sensitive tool to diagnose gallbladder perforation [12]. CT scan finding can be divided into primary gallbladder changes, pericholecystic changes and findings in extra-gallbladder organs. Primary gallbladder changes include wall thickening, wall enhancement, wall defect, intramural abscess, intramural gas, mural hemorrhage, presence of gallstones, bile duct stones or cystic duct stones, intraluminal membrane and intraluminal gas. Pericholecystic changes include pericholecystic fat stranding, pericholecystic fluid collection, abscess or biloma formation and presence of extra-luminal stones. Findings in organs other than the gallbladder consist of pericholecystic liver enhancement, liver abscess, portal vein thrombosis, lymphadenopathy, reactive mural thickening of adjacent hollow organs (hepatic flexure of colon and duodenum), intra-peritoneal free air, ascites and Mirizzi syndrome. The gallbladder perforation signs can be di- 
vided into direct and indirect signs: the demonstration of either calculi outside the gallbladder or a ruptured segment of the gallbladder wall is a direct indicator according to Pedosa et al [13]. Indirect indicators include the presence of an abscess outside the gallbladder and the presence of gallstones together with thickening of gallbladder wall. Sensitivity of CT scan in the detection of gallbladder perforation and biliary calculi has been reported to be between $88 \%$ and $89 \%$ [12].

Magnetic resonance imaging (MRI) may demonstrate the wall of the gallbladder and defects also. The biliary tree is better demonstrated by MRCP than other modalities in presence of gallbladder perforation in suspected cases of acute cholecystitis [14]. If USG and CT scans are not conclusive, the MRI is the modality of choice. However, the cost is the limiting factor.

In conclusion, to decrease the morbidity and mortality associated with gallbladder perforation, early diagnosis and surgical intervention are of prime importance. Presence of risk factors certainly warrants an aggressive investigation to rule out this serious complication. Gallbladder perforation should be considered in differential diagnosis in elderly patients presenting with peritonitis with an unknown etiology as was in this patient.

\section{Funding Sources}

None.

\section{Conflicts of Interest}

The authors have no conflicts of interest to disclose.

\section{References}

1. Roslyn J, Busuttil RW. Perforation of the gallbladder: a frequently mismanaged condition. Am J Surg. 1979;137(3):307-312.

2. Kalliafas S, Ziegler DW, Flancbaum L, Choban PS. Acute acalculous cholecystitis: incidence, risk factors, diagno- sis, and outcome. Am Surg. 1998;64(5):471-475.

3. Estevao-Costa J, Soares-Oliveira M, Lopes JM, Carvalho JL. Idiopathic perforation of the gallbladder: a novel differential diagnosis of acute abdomen. J Pediatr Gastroenterol Nutr. 2002;35(1):88-89.

4. Derici H, Kara C, Bozdag AD, Nazli O, Tansug T, Akca E. Diagnosis and treatment of gallbladder perforation. World J Gastroenterol. 2006;12(48):7832-7836.

5. Niemeier OW. Acute Free Perforation of the Gall-Bladder. Ann Surg. 1934;99(6):922-924.

6. Roslyn JJ, Thompson JE, Jr., Darvin H, DenBesten L. Risk factors for gallbladder perforation. Am J Gastroenterol. 1987;82(7):636-640.

7. Glenn F, MooreSW. Gangrene and perforation of the wall of the gallbladder. Arch Surg. 1942;44677-44686.

8. Chen JJ, Lin HH, Chiu CT, Lin DY. Gallbladder perforation with intrahepatic abscess formation. J Clin Ultrasound. 1990;18(1):43-45.

9. Gore RM, Ghahremani GG, Joseph AE, Nemcek AA, Jr., Marn CS, Vogelzang RL. Acquired malposition of the colon and gallbladder in patients with cirrhosis: CT findings and clinical implications. Radiology. 1989;171(3):739742 .

10. Sood BP, Kalra N, Gupta S, Sidhu R, Gulati M, Khandelwal N, Suri S. Role of sonography in the diagnosis of gallbladder perforation. J Clin Ultrasound. 2002;30(5):270274.

11. Solva M, Pamilo M, Paivansaio M, Taavitsainen M, Suramo L. Ultasonography in acute gallbladder perforation. Acta Radiol. 1988;2941-2944.

12. Tsai MJ, Chen JD, Tiu CM, Chou YH, Hu SC, Chang CY. Can acute cholecystitis with gallbladder perforation be detected preoperatively by computed tomography in ED? Correlation with clinical data and computed tomography features. Am J Emerg Med. 2009;27(5):574-581.

13. Pedrosa CS, Casanova R, Rodriguez R. CT findings in subacute perforation of the gallbladder: report on 5 cases. Eur J Radiol. 1981;1(2):137-142.

14. Sood B, Jain M, Khandelwal N, Singh P, Suri S. MRI of perforated gall bladder. Australas Radiol. 2002;46(4):438440. 